\title{
Aortic arch shape deformation after coarctation surgery: Effect on blood pressure response
}

\author{
Phalla Ou, MD, ${ }^{\mathrm{a}, \mathrm{b}}$ Elie Mousseaux, MD, PhD, ${ }^{\mathrm{c}}$ David S. Celermajer, MBBS, DSc, FRACP, ${ }^{\mathrm{d}}$ Elisa Pedroni, MD, ${ }^{\mathrm{e}}$ \\ Pascal Vouhe, MD, PhD, ${ }^{f}$ Daniel Sidi, MD, PhD, ${ }^{e}$ and Damien Bonnet, MD, $\mathrm{PhD}^{\mathrm{e}, \mathrm{g}}$
}

From INSERM UMR S678, Faculté de Médecine Pitié Salpétrière, ${ }^{a}$ Paris, France; the Departments of Pediatric Radiology, Pediatric Cardiology, ${ }^{\mathrm{e}}$ and Pediatric Cardiovascular Surgery, ${ }^{\mathrm{f}}$ Hôpital Necker-Enfants Malades, AP-HP, Paris, France; the Department of Cardiovascular Radiology, ${ }^{\mathrm{C}}$ Hôpital Européen Georges Pompidou, AP-HP, France; the Department of Medicine, ${ }^{\mathrm{d}}$ University of Sydney, Sydney, Australia; and INSERM E0016, Faculté de Médecine Necker-Enfants Malades, ${ }^{\mathrm{g}}$ Paris, France.

Supported by a grant from the Fédération Française de Cardiologie and from the Fondation Wyeth pour la Santé de l'Enfant et de l'Adolescent, Paris, France.

Received for publication March 4, 2006; revisions received May 15, 2006; accepted for publication May 30, 2006.

Address for reprints: Phalla Ou, MD, Department of Pediatric Radiology, Hôpital Necker-Enfants Malades, 149, rue de Sèvres 75743 Paris Cedex 15, France (E-mail: phalla.ou@nck.ap-hop-paris.fr).

J Thorac Cardiovasc Surg 2006;132:1105-11 0022-5223/ $\$ 32.00$

Copyright (c) 2006 by The American Association for Thoracic Surgery

doi:10.1016/j.jtcvs.2006.05.061
Objective: We sought to investigate the effect of particular deformations of aortic arch shape on blood pressure response in children who had undergone successful repair of coarctation of the aorta.

Methods: We studied normotensive patients (aged $15.1 \pm 5.8$ years) who had undergone coarctation surgery by means of simple $(n=43)$ or extended $(n=32)$ end-to-end anastomosis. All patients underwent systematic exercise testing during follow-up, as well as magnetic resonance imaging. Three types of aortic arch geometry were evident on magnetic resonance imaging: gothic arch with angular geometry and an increased height/width ratio of the arch, crenel arch with a rectangular shape, and normal arch with a smooth rounded shape. For each subject's arch, the continuous variable height/width ratio was calculated.

Results: Exercise-induced hypertension was more frequent in patients with gothic arch, with a prevalence of $83 \%$ at 15 years after surgical intervention $(95 \%$ confidence interval, $69 \%-97 \%$ ) versus $25 \%$ (95\% confidence interval, $0 \%-50 \%$ ) in those with crenel and 21\% (95\% confidence interval, 10\%-46\%) in those with normal arch geometry $(P<.001)$. The cumulative incidence of exercise-induced hypertension as a function of aortic arch geometry was significantly higher in patients with gothic arch geometry throughout follow-up. On multivariate analysis, both gothic arch geometry and higher height/width ratio were significantly correlated with exercise-induced hypertension.

Conclusion: An angulated gothic arch is independently associated with abnormal blood pressure response. This deformation of the aortic arch identifies a subgroup of subjects with postoperative coarctation at high risk of hypertension in young adult life.

$\mathrm{S}$ ystematic hypertension is a common complication after anatomically successful repair of coarctation of the aorta (CoA) in $20 \%$ to $40 \%$ of young adult survivors. ${ }^{1}$ Although hypertension might be somewhat less frequent with repair in early life, both resting and exercise-related or ambulatory hypertension are still common, ${ }^{2}$ particularly in studies with a follow-up of greater than 15 years. Furthermore, late hypertension is clearly a major risk factor for premature cardiovascular morbidity and mortality after CoA repair. ${ }^{1-3}$

Exaggerated blood pressure (BP) response to exercise is independently associated with increased risk of future hypertension in the general population ${ }^{4-7}$ and in normotensive individuals having risk factors for hypertension, ${ }^{8}$ including patients who had CoA repair in infancy. ${ }^{9}$ In subjects after CoA repair, this often occurs in the absence of recoarctation or aortic arch hypoplasia. ${ }^{3}$ The mechanisms of exerciseinduced or resting hypertension in patients who do not harbor these residual abnormalities are still uncertain. ${ }^{1}$

Few studies have focused on the role of aortic arch shape abnormalities on BP response. Recently, Roos-Hesselink and colleagues ${ }^{10}$ reported a striking frequency 


\author{
Abbreviations and Acronyms \\ $\mathrm{BP}=$ blood pressure \\ CI = confidence interval \\ $\mathrm{CoA}=$ coarctation of the aorta \\ $\mathrm{H} / \mathrm{W}=$ height $/$ width \\ MRI = magnetic resonance imaging \\ $\mathrm{RS}=$ residual stenosis
}

of abnormal aortic arch geometry late after CoA repair, but these were poorly characterized before the era of magnetic resonance imaging (MRI). Similarly, using MRI, we have demonstrated important modifications in the aortic arch anatomy in patients with successful CoA repair (ie, in the absence of recoarctation, aortic arch hypoplasia, or both). ${ }^{11}$ In this latter study we demonstrated that a peculiar gothic arch, so-called because of a particular postoperative deformation of the aortic arch with an angular shape and abnormally increased height/width $(\mathrm{H} / \mathrm{W})$ ratio of the thoracic aorta, as shown in Figure 1, $A$, was independently associated with resting hypertension. The question now arises as to whether exercise-induced hypertension is associated with the same abnormalities of aortic arch shape in this specific population with successful CoA repair.

The aim of the present study was to investigate whether exercise-induced hypertension in successfully repaired patients after coarctectomy is associated with abnormal aortic arch geometry. If so, this might help to identify a population of adolescents or young adults at high risk for hypertension and its complications late after CoA repair.

\section{Methods}

\section{Patients}

The study population (Table 1) comprised 75 patients with CoA repair, of whom 25 were included from a previous study. ${ }^{11}$ All patients had undergone surgical repair before 3 months of age; 58 (77.3\%) infants were less than 1 month old at the time of surgical intervention. The mean \pm standard deviation weight was $3.1 \pm 0.7$ $\mathrm{kg}$ at the time of the operation.

The operation was performed through a standard left posterolateral thoracotomy. The technique of repair was chosen according to the presence or absence of hypoplasia of the aortic arch. Standard end-to-end anastomosis $(\mathrm{n}=43$ [57.3\%]; median age at surgical intervention, 0.3 months) was performed when CoA was simple and without hypoplasia of the aortic arch. Extended endto-end anastomosis $(\mathrm{n}=32$ [42.7\%]; median age at surgical intervention, 0.28 months) was performed as described previously $^{12}$ when CoA was associated with hypoplasia of the aortic arch. In all cases the aortic arch was repaired with interrupted and absorbable sutures.

Consecutively eligible subjects were recruited between 2003 and 2005. Criteria for inclusion were (1) systolic and diastolic BP at rest within the normal range, according to the Task Force Report on High Blood Pressure in Children and Adolescents ${ }^{13}$; (2) no
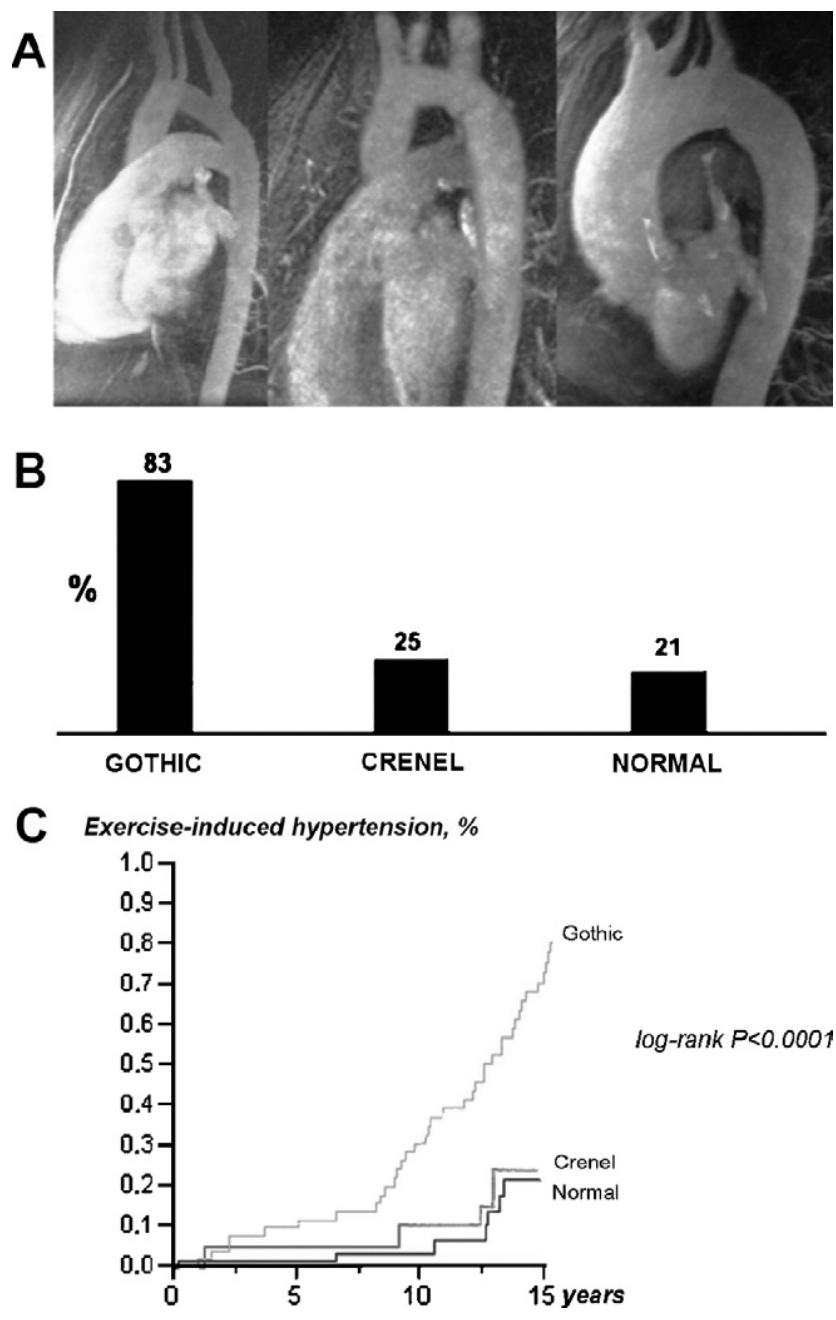

Figure 1. Relation between aortic arch shape and blood pressure response. A, Three types of aortic arch morphology after coarctation repair defined on the basis of MRI. Left to right, Gothic, crenel, and normal geometry. B, Percentage of patients with exercise-induced hypertension at $\mathbf{1 5}$ years' follow-up in the $\mathbf{3}$ types of aortic arch geometry. C, Cumulative incidence of exerciseinduced hypertension as a function of aortic arch geometry with Kaplan-Meier curves.

significant associated cardiac anomalies; (3) no cardiac medication; and (4) absence of clinical and Doppler flow evidence of structural restenosis in the aorta, with a right arm-leg systolic BP gradient at rest of less than $15 \mathrm{~mm} \mathrm{Hg}$ and a systolic peak flow velocity of less than $3 \mathrm{~m} / \mathrm{s}$ in the descending aorta with no evidence of diastolic runoff on Doppler echocardiography. Consistent with this, all subjects had unobstructed aortic arches on MRI $(<30 \%$ narrowing at the minimum lumen diameter, see below). We excluded patients who had undergone balloon dilatation or stent placement and those who had undergone any intervention for recoarctation.

The hospital ethics committee approved this study, and all patients or their guardian provided informed consent. 
TABLE 1. Population characteristics $(n=75)$

\begin{tabular}{|c|c|c|c|}
\hline & Mean \pm SD & Median & Range \\
\hline \multicolumn{4}{|l|}{ Demography } \\
\hline $\mathrm{M} / \mathrm{F}$ & $49 / 26$ & & \\
\hline Age (y) & $15.1 \pm 5.8$ & 14.5 & $6-33.5$ \\
\hline \multicolumn{4}{|l|}{ Anthropometry } \\
\hline Weight (kg) & $56.6 \pm 16.5$ & 55 & $19-80$ \\
\hline Height $(\mathrm{cm})$ & $165 \pm 21$ & 163 & $105-187$ \\
\hline \multicolumn{4}{|l|}{$\mathrm{BP}$ at rest (upper right $\operatorname{limb}$ ) } \\
\hline Systolic BP (mm Hg) & $117 \pm 12$ & 117 & $86-137$ \\
\hline Diastolic BP (mm Hg) & $60 \pm 11$ & 58 & $38-80$ \\
\hline \multicolumn{4}{|l|}{ Operation } \\
\hline Age at operation (mo) & $0.27 \pm 0.2$ & 0.3 & $0.1-168$ \\
\hline Duration of follow-up (y) & $15 \pm 6.5$ & 15.0 & $1-30$ \\
\hline
\end{tabular}

$S D$, Standard deviation; $M / F$, male/female; $B P$, blood pressure.

BP Measurements, Rest, and Exercise

Resting BP in the right arm and leg and resting arm-leg systolic BP gradient were measured simultaneously by using the automatic oscillometric method (Accutor 4; Datascope Corp, Montvale, NJ) with appropriately sized cuffs that recovered at least two thirds of the upper arm and of the calf after at least 5 minutes of rest in the supine position. The average of the second and third measurements was used in the analysis.

In our institution treadmill exercise testing is routinely and systematically performed in all children who have had successful CoA repair: the first test is performed at age 8 years and then every 2 years thereafter. Exercise treadmill testing was performed according to the Bruce protocol, in which the speed and slope of a treadmill was increased every 3 minutes. ${ }^{14}$ During the last minute of each exercise stage, systolic BP was measured with a mercury column sphygmomanometer. Subjects exercised until reaching an age-specific target heart rate or the development of symptoms leading to termination of the test. Systolic hypertension at exercise was defined in children as maximum systolic BP at the 95th or greater percentile for a separate reference population ${ }^{5,14}$ or 210 $\mathrm{mm} \mathrm{Hg}$ or greater in men and $190 \mathrm{~mm} \mathrm{Hg}$ or greater in women. ${ }^{5,15}$ Group HT included the patients with exercise hypertension, and group $\mathrm{N}$ included those who had a normal $\mathrm{BP}$ response to peak exercise.

\section{MRI Study}

Aortic arch geometry measurements were made by an experienced observer who was unaware of all BP measurements (1.5-T magnet with $23 \mathrm{mT} / \mathrm{m}$ gradients; Signa LX; GE Medical Systems, Milwaukee, Wis). As previously described, ${ }^{11}$ we performed both qualitative and quantitative measurements for characterizing postcoarctectomy aortic arch shape. First, morphology of the aortic shape was classified in 3 categories based on the global geometry of the aortic arch in the left anterior oblique projection: gothic, crenel, and normal (Figure 1, A). Briefly, gothic arch had a triangular form, crenel arch had a rectangular form, and normal arch had a semicircular form. Second, 3 quantitative measurements were made, including maximal height and width of the aortic arch and the degree of residual stenosis (RS), as follows: $\mathrm{RS}=100 \mathrm{x}$ $(1-[\varnothing \mathrm{m} / \varnothing \mathrm{D}])$, where $\emptyset \mathrm{m}$ is the smallest diameter at the anas-

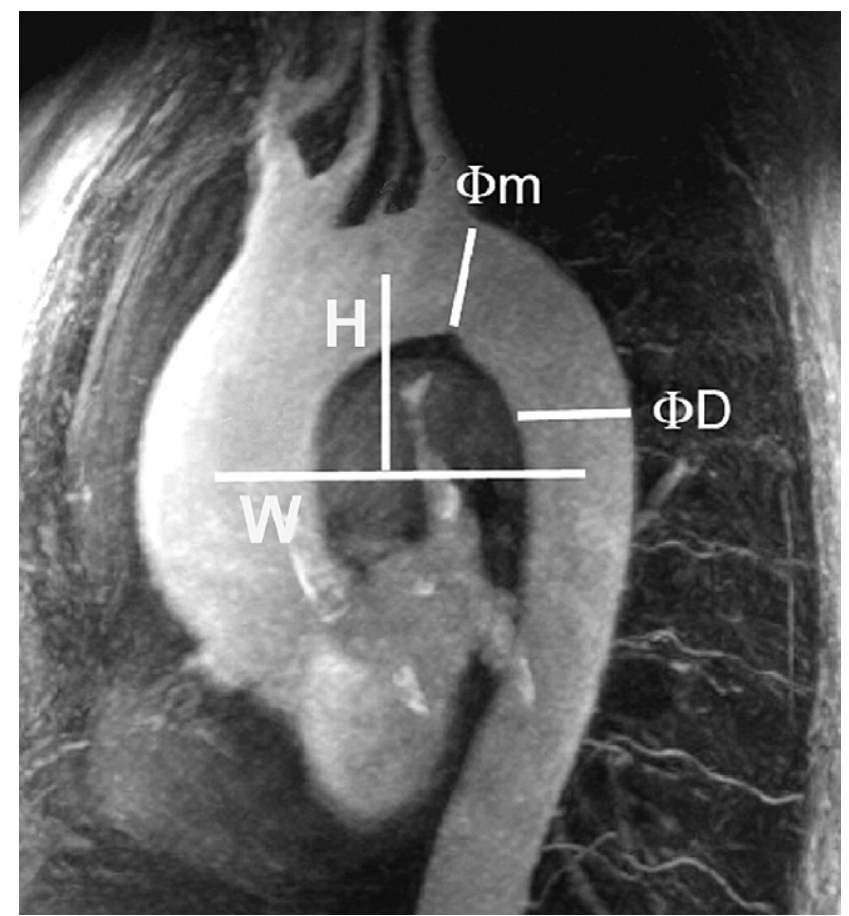

Figure 2. Aortic shape measurements on MRI transverse width of the aortic arch (W) was measured as the maximal horizontal distance between the midpoints of the ascending and descending aorta close to the axial plane going through the right pulmonary artery. Height of the aortic arch $(H)$ was measured as the maximal vertical distance between $W$ and the highest midpoint of the aortic arch. $\emptyset_{m}$ is the smallest diameter at the anastomosis, and $\varnothing D$ is the diameter of the descending aorta $10 \mathrm{~cm}$ from the anastomosis.

tomosis and $\varnothing \mathrm{D}$ is the diameter of the descending aorta $10 \mathrm{~cm}$ distal to the anastomosis (Figure 2).

\section{Statistical Analysis}

All data were analyzed with the StatView SE software and the R software Version 1.7.0. The normality Kolmogorov-Smirnov test was performed to determine whether continuous variables were normally distributed.

Data are presented as the mean value \pm standard deviation or median with range when appropriate. Univariate comparisons of continuous variables were performed by using the Student $t$ test or the Wilcoxon rank sum test when the normality assumption was not satisfied. Categoric variables were compared by using the $\chi^{2}$ test.

Aortic arch geometry was characterized as both a categoric (3 categories: gothic, crenel, or normal) and a continuous (H/W ratio) variable. The $\mathrm{BP}$ response to exercise was treated also as both a categoric (HT or N status) and continuous (level of systolic BP at peak exercise) variable. The relation between aortic arch geometry and the incidence of exercise-induced hypertension over the follow-up period was evaluated by using Kaplan-Meier survival 
TABLE 2. Clinical and geometric variables in the 3 subtypes of aortic arch geometry

\begin{tabular}{|c|c|c|c|c|c|c|c|}
\hline & \multirow{2}{*}{$\begin{array}{l}\text { Gothic } \\
(n=30)\end{array}$} & \multirow{2}{*}{$\begin{array}{l}\text { Crenel } \\
(\mathrm{n}=12)\end{array}$} & \multirow{2}{*}{$\begin{array}{l}\text { Normal } \\
(\mathrm{n}=33)\end{array}$} & \multirow{2}{*}{$\begin{array}{l}P \text { value for } \\
\text { ANOVA }\end{array}$} & \multicolumn{3}{|c|}{$P$ value } \\
\hline & & & & & Gothic/normal & Gothic/crenel & Crenel/normal \\
\hline Age (y) & $16 \pm 5.9$ & $14.8 \pm 6.7$ & $14.6 \pm 5.2$ & .022 & .017 & .29 & .51 \\
\hline Weight (kg) & $63 \pm 14.3$ & $58.1 \pm 12.3$ & $58.3 \pm 16.6$ & .008 & .019 & .0005 & .32 \\
\hline Height $(\mathrm{cm})$ & $170 \pm 16$ & $167 \pm 18$ & $168 \pm 23$ & .017 & .0045 & .0086 & .9 \\
\hline Age at operation, median (mo) & 0.34 & 0.27 & 0.32 & .5 & .7 & .9 & .8 \\
\hline $\begin{array}{l}\text { Extended end-to-end } \\
\text { anastomosis }(\%)\end{array}$ & 43 & 42 & 42 & .8 & .7 & .5 & .7 \\
\hline \multicolumn{7}{|l|}{$\mathrm{BP}$ at rest } & .88 \\
\hline Systolic BP (mm Hg) & $124.1 \pm 11.9$ & $115 \pm 12.2$ & $112.4 \pm 14.7$ & .003 & .001 & .03 & .59 \\
\hline Diastolic BP (mm Hg) & $75.5 \pm 5.7$ & $73.3 \pm 7.5$ & $72.3 \pm 6.3$ & .66 & .06 & .32 & .65 \\
\hline $\begin{array}{l}\text { Arm-leg systolic BP } \\
\text { gradient (mm Hg) }\end{array}$ & $2 \pm 8.8$ & $0.1 \pm 6.2$ & $-0.9 \pm 10.9$ & .2 & .25 & .50 & .76 \\
\hline \multicolumn{8}{|l|}{ BP at peak exercise } \\
\hline Systolic BP (mm Hg) & $210.2 \pm 33.1$ & $171.2 \pm 25.9$ & $177.4 \pm 33.6$ & .0002 & .0002 & .0008 & .56 \\
\hline Diastolic BP (mm Hg) & $74.5 \pm 6.3$ & $72.5 \pm 7.2$ & $72.2 \pm 6.6$ & .56 & .16 & .38 & .89 \\
\hline \multicolumn{8}{|l|}{ Geometric parameter } \\
\hline H/W ratio & $0.8 \pm 0.16$ & $0.69 \pm 0.14$ & $0.65 \pm 0.15$ & $<.0001$ & $<.0001$ & .07 & .48 \\
\hline
\end{tabular}

ANOVA, Analysis of variance; $B P$, blood pressure; $H / W$, height/width.

curves. Multivariate analyses were also used to determine prognostic factors for hypertension at peak exercise. The following variables were included in the models: age at time of the study, duration of follow-up, height, weight, resting arm-leg systolic BP gradient, RS, H/W, and aortic arch geometry category. First, we studied hypertensive status (group HT or group N) as a response variable by using multiple logistic regression analysis models. Goodness of fit of these models was checked by using the Le Cessie-van Houwelingen-Copas-Hosmer test. When multiple comparisons were performed, we used the Bonferroni method to compute the adjusted $P$ values. Second, we studied the level of systolic BP as a response variable by using multiple linear regression or analyses of covariance when appropriate. The linearity assumption was assessed graphically for the continuous independent variables. We checked before normality and homogeneity of variance of the residuals with plots of residuals against fitted value and histograms. When multiple comparisons were performed, we used the Tukey method to compute adjusted $P$ values.

\section{Results}

\section{Aortic Arch Geometry}

The aortic arch was abnormal in 42 (56\%) patients; 30 (40\%) patients had gothic geometry, and $12(16 \%)$ had crenel geometry. The remaining 33 (44\%) subjects had normal geometry. $\mathrm{H} / \mathrm{W}$ ratio was significantly higher in patients with a gothic geometry $(\mathrm{H} / \mathrm{W}$ ratio, $0.8 \pm 0.16)$ when compared with the 2 others morphologies $(P<$ .0001). There were no differences among the 3 geometries concerning RS, which was less than $30 \%$ in all cases. As shown in Table 2, the proportion of subjects with extended end-to-end (vs simple end-to-end) anastomoses was similar between groups.

\section{Correlation Between Aortic Arch Geometry and Blood Pressure}

Thirty-five (46.7\%) patients were hypertensive during exercise. Hypertension was more frequent in patients with gothic arch geometry (25/30 [83\%]; 95\% confidence interval $[\mathrm{CI}], 69 \%-97 \%)$ than in the crenel form $(3 / 12$ [25\%]; 95\% CI, 0\%-50\%) and the normal form (7/33 [21\%]; 95\% CI, $10 \%-46 \% ; P<.001$; Figure $1, B)$. The cumulative incidence of exercise-induced hypertension as a function of the 3 categories of aortic arch geometry is shown in Figure 1, $C$. The curves demonstrated that the subjects with gothic arch geometry had the highest incidence of exerciseinduced hypertension compared with those with crenel and normal geometry (log-rank test, $P<.0001)$.

On univariate analysis, age at the time of the study, duration of follow-up, weight, height, systolic BP at rest and at peak exercise, and $\mathrm{H} / \mathrm{W}$ ratio were significantly higher in the HT group (Table 3). By using multiple logistic regression analysis, independent factors associated with hypertension status were both gothic arch geometry and H/W ratio $(P=.009$ and $P=.038$, respectively) when the independent variables used in the model were age at time of the study, duration of follow-up, weight, height, resting arm-leg systolic BP gradient, RS, and aortic arch geometry category or $\mathrm{H} / \mathrm{W}$ ratio.

We also compared the level of systolic BP at peak exercise among the 3 geometries of the aortic arch. As shown in Table 2, systolic BP at peak exercise was significantly higher in patients with gothic arch geometry $(P=$ .0002). On univariate regression analysis, there was a sig- 
TABLE 3. Comparison of clinical and geometric variables in the $\mathrm{HT}$ and $\mathbf{N}$ groups

\begin{tabular}{lccc}
\hline & HT & N & P value \\
\hline $\mathrm{n}$ (total = 75) & 35 & 40 & \\
Age (y) & $14.9 \pm 4$ & $13.7 \pm 5$ & .0001 \\
Weight (kg) & $59 \pm 12$ & $52 \pm 11$ & $<.0001$ \\
Height (cm) & $170 \pm 12$ & $163 \pm 13$ & $<.0001$ \\
Age at operation, & 0.3 & 0.29 & .6 \\
$\quad$ median (mo) & & & \\
Duration of follow-up (y) & $14.6 \pm 6$ & $14.2 \pm 5$ & .04 \\
BP at rest & & & \\
$\quad$ Systolic BP (mm Hg) & $125.9 \pm 10.8$ & $110.1 \pm 12.6$ & $<.0001$ \\
$\quad$ Diastolic BP (mm Hg) & $75.7 \pm 6.1$ & $72 \pm 6.2$ & .01 \\
Arm-leg systolic BP & $2.4 \pm 7.9$ & $-1.3 \pm 10.5$ & .09 \\
$\quad$ & & & \\
gradient (mm Hg) & & & \\
$\quad$ Systolic BP (mm Hg) & $220.1 \pm 26.4$ & $162.7 \pm 17.3$ & $<.0001$ \\
$\quad$ Diastolic BP (mm Hg) & $74.9 \pm 6.6$ & $71.6 \pm 6.3$ & .036 \\
\hline BP, Blood pressur. & & & \\
\hline
\end{tabular}

$B P$, Blood pressure.

nificant correlation between systolic BP at peak exercise and age at the time of the operation, duration of follow-up, height, weight, and H/W ratio but not with arm-leg systolic BP gradient or RS. By using analysis of covariance or multiple linear regression analysis with the same independent variables, only gothic arch geometry and $\mathrm{H} / \mathrm{W}$ ratio were correlated with systolic BP at peak exercise $(P=.04$ and $P=.02$, respectively).

\section{Discussion}

Long-term follow-up studies have shown that systemic hypertension is a common and important complication in patients who have had CoA repair in infancy. In addition, life expectancy in this population is significantly shorter than that of a normal population, largely because of cardiovascular and cerebrovascular events, most frequently related to hypertension. ${ }^{1-3}$ The prevalence of hypertension increases with length of follow-up after CoA repair, suggesting that the risk factors for new-onset hypertension are present very early in life or that they might appear progressively with growth. ${ }^{1-3}$ Early detection of hypertension and identification of risk factors in patients who have undergone CoA repair is thus a crucial issue to reduce the incidence of late cardiovascular events. Exaggerated BP response during exercise is generally predictive of resting hypertension, ${ }^{4-8}$ and MRI can be used to accurately diagnose abnormalities of the postoperative aortic arch..$^{10,11} \mathrm{We}$ have previously reported that resting hypertension after CoA repair was associated with a gothic geometry of the aortic arch. ${ }^{11}$ We now demonstrate that this particular gothic arch geometry is strongly associated with exerciseinduced hypertension.

\section{Exercise Testing After CoA Repair}

The prevalence of higher than expected peak systolic BP responses, diastolic BP responses, or both on exercise is low in healthy populations (3\%-12\%), ${ }^{16-18}$ suggesting that exercise testing would not be useful in detecting hypertension in the general population. However, exercise testing is much more relevant in patients at high risk for hypertension, such as those who have had repair of CoA. In addition, patients with gothic arch geometry had significantly higher resting systolic BP (but still within the normal range, Table 2), and it has been previously shown that subjects with high-normal resting BP who exhibited an exaggerated exercise BP response were more likely to have resting hypertension in the future. ${ }^{8}$ Thus our patients with gothic arch geometry exhibit well-known risk factors for future hypertension, with highnormal resting systolic BP and an exaggerated BP response to exercise.

The usefulness of exercise testing has been questioned in follow-up after CoA repair. Recently, Swan and coworkers ${ }^{19}$ discouraged the use of exercise testing, arguing that it has been shown to be insufficient to predict recoarctation. In contrast, our study demonstrates that exercise testing is still a valuable tool in the surveillance of patients who have undergone CoA repair. Indeed, we now demonstrate that exercise testing, when coupled with MRI, is helpful in screening patients at risk of hypertension, namely those who presented with abnormal BP responses during exercise, abnormal aortic arch shape on MRI, or both.

\section{Aortic Arch Shape and Mechanisms of Exaggerated Systolic BP}

The pathogenesis whereby either the preoperative anatomy or the technique of surgical repair might result in gothic arch geometry is unclear, although it does not appear related to arch hypoplasia or the surgical technique of extended end-to-end anastomosis. This geometric pattern appears to arise from an acute angulation in the arch associated with a greater growth in the height compared with the width of the thoracic aorta. We speculate that an abnormally short aortic isthmus, that portion of the aorta between the left subclavian artery and the ductus arteriosus, might predispose to acute angulation and gothic arch geometry after repair; however, this will need to be tested prospectively in a study in which detailed preoperative measures of the length of the isthmus can be made, optimally by means of MRI. It is also unclear whether surgical techniques of CoA repair might be modifiable, such that normal arch anatomy can be fashioned in which the risk of an angulated gothic arch is thought to be high. It is likely that aortoplasty with use of a patch might facilitate the construction of a smooth and rounded aortic arch; however, we would obviously not recommend this technique because it is associated with a high incidence of late aneurysm formation at the repair site. ${ }^{20}$ 
The pathophysiologic mechanism of exercise-induced hypertension has not been clearly defined in patients with successful CoA repair. Our findings could contribute, at least in part, to explaining the complex mechanisms of abnormal BP response in patients after coarctectomy. Indeed, gothic geometry in our patients could lead to flow disturbances at the point of angulation, including early return of the incident pressure wave that would be expected to increase systolic BP and pulse pressure. Such anomalies might resemble the systolic wave reflections observed with aging and in essential hypertension. This could contribute to increased central aortic stiffness and upper-limb hypertension in patients with gothic geometry. These hypotheses need to be tested by means of further investigation.

It is noteworthy that some subjects in the HT group do not have gothic arch geometry but have normal or crenel geometry; thus angulation of the aortic arch does not exclusively explain hypertension after CoA repair. Recently, Vriend and associates ${ }^{21}$ showed that even a mild residual narrowing at the site of CoA repair was a strong and independent predictor of daytime systolic BP and common carotid artery wall thickening. The same mechanism of early wave reflection could account for these results. These authors raised the question of lowering the threshold for reintervention in mild recoarctation to improve long-term outcome in such patients.

\section{Clinical Implications}

Our results might have implications for surgical and medical treatment of CoA. It is possible that identification of the risk of hypertension associated with an angulated gothic arch might inform surgical strategies toward techniques that might result in a more rounded and smooth arch geometry (although patch aortoplasty should not be considered, as noted above). Even if surgical techniques cannot be modified in this way, for practical reasons, our study identifies a high-risk group of subjects with $\mathrm{CoA}$ who require particularly vigilant surveillance and perhaps even early medical intervention. Indeed, we now recommend regular exercise testing and MRI in the surveillance of patients who have undergone $\mathrm{CoA}$ repair, considering these 2 examinations useful for the early identification of young patients at high risk for hypertension and its cardiovascular complications.

\section{Limitations}

By observing the magnetic resonance images of our postoperative subjects with $\mathrm{CoA}$, we were initially cognizant of 3 recognizable categories of arch geometry: an angulated (gothic) form, a rectangular (crenel) form, and a smooth rounded (normal) arch. For example, the gothic arch depicted in Figure 1, $A$, was easy to identify. Nevertheless, this qualitative classification of the aortic arch geometry might be difficult in patients who present with intermediate geom- etries. For this reason, we have also calculated the more objective $\mathrm{H} / \mathrm{W}$ ratio for characterizing aortic arch geometry. In our study increases in $\mathrm{H} / \mathrm{W}$ ratio were also predictive of exercise-induced hypertension, as well as gothic arch geometry as a categoric variable.

The relation between resting hypertension and aortic arch geometry after CoA repair has already been described previously by our group ${ }^{11}$; the current findings extend the relevance of the previous findings to the important situation of exercise-induced hypertension. Our message is that the main criteria for considering a coarctation as successfully repaired should include both absence of RS and absence of aortic arch deformation.

\section{Conclusion}

In patients with an anatomically successful CoA repair, exercise-induced hypertension is associated with gothic geometry of the aortic arch and an increased $\mathrm{H} / \mathrm{W}$ ratio of the thoracic aorta. Patients with this angular geometry are at high risk of resting hypertension, and thus careful long-term surveillance of these patients is mandatory. Our results might interest surgeons by raising technical questions of prevention of aortic arch deformation.

\section{References}

1. Celermajer DS, Greaves K. Survivors of coarctation repair: fixed but not cured. Heart. 2002;88:113-4.

2. de Divitiis M, Rubba P, Calabro R. Arterial hypertension and cardiovascular prognosis after successful repair of aortic coarctation: a clinical model for the study of vascular function. Nutr Metab Cardiovasc Dis. 2005; 15:382-94

3. Cohen M, Fuster V, Steele PM, Driscoll D, McGoon DC. Coarctation of the aorta. Long-term follow-up and prediction of outcome after surgical correction. Circulation. 1989;80:840-5.

4. Matthews CE, Pate RR, Jackson KL, Ward DS, Macera CA, Kohl HW, et al. Exaggerated blood pressure response to dynamic exercise and risk of future hypertension. J Clin Epidemiol. 1998;51:29-35.

5. Manolio TA, Burke GL, Savage PJ, Sidney S, Gardin JM, Oberman A Exercise blood pressure response and 5-year risk of elevated blood pressure in a cohort of young adults: the CARDIA study. Am J Hypertens. 1994;7:234-41.

6. Singh JP, Larson MG, Manolio TA, O'Donnell CJ, Lauer M, Evans $\mathrm{JC}$, et al. Blood pressure response during treadmill testing as a risk factor for new-onset hypertension. The Framingham Heart Study. Circulation. 1999;99:1831-6.

7. Tsumura K, Hayashi T, Hamada C, Endo G, Fujii S, Okada K. Blood pressure response after two-step exercise as a powerful predictor of hypertension: the Osaka Health Survey. J Hypertens. 2002;20:1507-12.

8. Miyai N, Arita M, Morioka I, Miyashita K, Nishio I, Takeda S. Exercise BP response in subjects with high-normal BP: exaggerated blood pressure response to exercise and risk of future hypertension in subjects with high-normal blood pressure. J Am Coll Cardiol. 2000; 36:1626-31.

9. Vriend JW, van Montfrans GA, Romkes HH, Vliegen HW, Veen G, Tijssen JG, et al. Relation between exercise-induced hypertension and sustained hypertension in adult patients after successful repair of aortic coarctation. J Hypertens. 2004;22:501-9.

10. Roos-Hesselink JW, Scholzel BE, Heijdra RJ, Spitaels SE, Meijboom FJ, Boersma E, et al. Aortic valve and aortic arch pathology after coarctation repair. Heart. 2003;89:1074-7. 
11. Ou P, Bonnet D, Auriacombe L, Pedroni E, Balleux F, Sidi D, et al. Late systemic hypertension and aortic arch geometry after successful repair of coarctation of the aorta. Eur Heart J. 2004;25:1853-9.

12. Vouhe PR, Trinquet F, Lecompte Y, Vernant F, Roux PM, Touati G, et al. Aortic coarctation with hypoplastic aortic arch. Results of extended end-to-end aortic arch anastomosis. J Thorac Cardiovasc Surg. 1988;96:557-63.

13. National High Blood Pressure Education Program Working Group on Hypertension Control in Children and Adolescents. Update on the 1987 Task Force Report on High Blood Pressure in Children and Adolescents: a working group report from the National High Blood Pressure Education Program. Pediatrics. 1996;98:649-58.

14. Ellestad MH. Stress testing: principles and practice. 3rd ed. Philadelphia: FA Davis; 1986.

15. Lauer MS, Levy D, Anderson KM, Plehn JF. Is there a relationship between exercise systolic blood pressure response and left ventricular mass? The Framingham Heart Study. Ann Intern Med. 1992;116:203-10.
16. Svardsudd K, Wedel H, Wilhelmsen L. Factors associated with the initial blood pressure level and with the subsequent blood pressure increase in a longitudinal population study. The study of men born in 1913. Eur Heart J. 1980;1:345-54.

17. Wilson NV, Meyer BM. Early prediction of hypertension using exercise blood pressure. Prev Med. 1981;10:62-8.

18. Mausner JS, Kramer S. In: Mausner JS, Bahn AK, editors. Epidemiology—an introduction text. Philadelphia: WB Saunders; 1985.

19. Swan L, Goyal S, Hsia C, Hechter S, Webb G, Gatzoulis MA. Exercise systolic blood pressures are of questionable value in the assessment of the adult with a previous coarctation repair. Heart. 2003;89:189-92.

20. Serfontein SJ, Kron IL. Complications of coarctation repair. Semin Thorac Cardiovasc Surg Pediatr Card Surg Annu. 2002;5:206-11.

21. Vriend JW, Zwinderman AH, de Groot E, Kastelein JJ, Bouma BJ, Mulder BJ. Predictive value of mild, residual descending aortic narrowing for blood pressure and vascular damage in patients after repair of aortic coarctation. Eur Heart J. 2005;26:84-90. 